\title{
SECOND ORDER LINEARIZED THEORY OF FREE-SURFACE FLOW IN POROUS MEDIA
}

A certain analogy exists between the equation of irrotational motion of an ideal liquid bounded by a freesurface and the free-surface flow of a liquid in a porous medium, obeying Darcy's Law.

In both cases the velocity potential is a harmonic function; on the free-surface the kinematic condition is identical. The dynamic one is different: the Bernoulli relation yields equations of a hyperbolic nature (conservative motion) while Darcy's Law yields parabolic equations (dissipative motion).

In both cases the nonlinearity of the potential boundary condition on the free-surface constitutes the main mathematical difficulty of the problem.

However, while in the theory of flow of an ideal liquid (especially water-wave theory) considerable progress has been achieved by the linearized theory, and approximations of first, second and higher order have been derived, only some first order solutions are known in the theory of flow through porous media.

The purpose of this paper is to derive the linearized equations of free-surface flow through porous media in a systematic manner, with some applications to the second order theory.

\section{The exact equations}

In this work the saturated flow of an incompressible, homogeneous liquid through an isotropic, homogeneous and non-deformable porous medium will be considered.

The exact equations of free-surface flow are already available in several sources (for example PolubarinovaKotchina [1952]), and are reproduced here for convenience of reference.

Let $\rho$ and $\gamma$ be the density and specific weight of the liquid respectively, $\mu$-its dynamic viscosity, $k$-the intrinsic hydraulic conductivity of the porous medium, $\mathrm{K}=(k \gamma / \mu)$ its hydraulic conductivity and $n$-its effective porosity coefficient.

With $\varphi=-\mathrm{K}[(\mathrm{P} / \gamma)+z]$ and $\eta(x, y, t)$, the velocity potential and the free-surface expression respectively, the following equations are satisfied:

in the field of flow:

$$
\nabla^{2} \varphi=\frac{\partial^{2} \varphi}{\partial x^{2}}+\frac{\partial^{2} \varphi}{\partial y^{2}}+\frac{\partial^{2} \varphi}{\partial z^{2}}=0
$$

$$
n \frac{\partial \varphi}{\partial t}+K \frac{\partial \varphi}{\partial z}+\left(\nabla \varphi^{2}\right) \equiv n \frac{\partial \varphi}{\partial t}+K \frac{\partial \varphi}{\partial z}+\left(\frac{\partial \varphi}{\partial x}\right)^{2}+\left(\frac{\partial \varphi}{\partial y}\right)^{2}+\left(\frac{\partial \varphi}{\partial z}\right)^{2}=0
$$

* Lecturer, Hydraulic Laboratory, Technion-Israel Institute of Technology. 


\section{G. DAGAN}

and:

on the free-surface $z=\eta(x, y, t)$.

$$
\eta=-\frac{1}{\mathrm{~K}} \varphi(x, y, z, t)
$$

On the other boundaries the more common conditions encountered are:

on an impervious boundary:

$$
\frac{\partial \varphi}{\partial n}=0
$$

$$
\varphi=\mathrm{const}
$$

on the boundary with liquid free of porous medium:

$$
\varphi=-\mathrm{K} z
$$

on a seepage face.

Exact solutions to these equations have been achieved only for steady bidimensional flow, mainly by the hodograph method (a brief review of this method is presented by Bear and Dagan [1962]).

in the flow region.

\section{The derivation of the linearized equations}

\section{by the approximation of small free-surface disturbances}

This approximation is analogous to that of infinitesimal-waves. The basic assumption is that both $\varphi$ and $\eta$ may be expanded in power series of a small parameter $\varepsilon$ :

$$
\begin{gathered}
\varphi(x, y, z, t)=\varphi_{0}(x, y, z)+\varepsilon \varphi_{1}(x, y, z, t)+\varepsilon^{2} \varphi_{2}(x, y, z, t)+\ldots \\
\eta(x, y, t)=\eta_{0}(x, y)+\varepsilon \eta_{1}(x, y, t)+\varepsilon^{2} \eta_{2}(x, y, t)+\ldots
\end{gathered}
$$

The nature of $\varepsilon$ is not specified; obviously, it has the character of a perturbation. $\varphi_{0}$ and $\eta_{0}$ represent the nonperturbed steady-state.

The substitution of $\varphi$ from (II.1) into (0.1) yields:

hence:

$$
\nabla^{2} \varphi=\nabla^{2} \varphi_{0}+\varepsilon \nabla^{2} \varphi_{1}+\varepsilon^{2} \nabla^{2} \varphi_{.2}+\ldots=0
$$

The condition to be satisfied on the free-surface will result from equations (I.2), (II.1) and (II.2).

First, the value of $\varphi$ and its derivatives on the free-surface may be derived by expansion on the surface $z=\eta_{0}(x, y)$ :

$\left.\varphi(x, y, z, t)\right|_{z=\eta}=\left.\varphi_{0}\right|_{z=\eta_{0}}+\varepsilon\left(\left.\eta_{1} \frac{\partial \varphi_{0}}{\partial z}\right|_{z=\eta_{0}}+\left.\varphi_{1}\right|_{z=\eta_{0}}\right)+\varepsilon^{2}\left(\left.\eta_{2} \frac{\partial \varphi_{0}}{\partial z}\right|_{z=\eta_{0}}+\left.\frac{\eta_{1}^{2}}{2} \frac{\partial^{2} \varphi_{01}}{\partial z^{2}}\right|_{z=\eta_{0}}+\left.\eta_{1} \frac{\partial \varphi_{1}}{\partial z}\right|_{z=\eta_{0}}+\left.\varphi_{2}\right|_{z=\eta_{0}}\right)+\ldots$

Hence, from (I.3) and (II.5) by identification:

$$
\begin{aligned}
& \frac{\partial^{2} \varphi_{0}}{\partial x^{2}}+\frac{\partial^{2} \varphi_{0}}{\partial y^{2}}+\frac{\partial^{2} \varphi_{0}}{\partial z^{2}}=0 \\
& \frac{\partial^{2} \varphi_{1}}{\partial x^{2}}+\frac{\partial^{2} \varphi_{1}}{\partial y^{2}}+\frac{\partial^{2} \varphi_{1}}{\partial z^{2}}=0
\end{aligned}
$$

$$
\begin{aligned}
& \eta_{0}=-\left.\frac{1}{\mathrm{~K}} \varphi_{0}\right|_{z=\eta_{0}} \\
& \eta_{1}=-\left.\frac{\varphi_{1}}{\mathrm{~K}+\left(\partial \varphi_{0} / \partial z\right)}\right|_{z=\eta_{0}} \\
& \eta_{2}=-\left.\frac{\eta_{1}\left(\frac{\eta_{1}}{2}-\frac{\partial^{2} \varphi_{0}}{\partial z^{2}}+\frac{\partial \varphi_{1}}{\partial z}\right)+\varphi_{2}}{\mathrm{~K}+\left(\partial \varphi_{0} / \partial z\right)}\right|_{z=\eta_{0}}
\end{aligned}
$$

Expanding $\varphi$ derivatives in a similar way, substituting in (I.2), it will result finally from (I.2), (II.4) and (II.6) for the different order terms:

$$
\begin{aligned}
& \nabla^{2} \varphi_{0}=0 \quad z<\eta_{0} \\
& \left.\begin{array}{rl}
\left(\nabla \varphi_{0}\right)^{2}+\mathrm{K} \frac{\partial \varphi_{0}}{\partial z} & =0 \\
\eta_{0} & =-\frac{\varphi_{0}}{\mathrm{~K}}
\end{array}\right\} \text { on } z=\eta_{0} \\
& \nabla^{2} \varphi_{1}=0 \quad z<\eta_{0}
\end{aligned}
$$




$$
\begin{aligned}
& \left.\left.n \frac{\partial \varphi_{1}}{\partial t}+2 \nabla \varphi_{0} \cdot \nabla \varphi_{1}+\eta_{1} \frac{\partial}{\partial z}\left(\nabla \varphi_{0}\right)^{2}+\mathrm{K}\left(\eta_{1} \frac{\partial^{2} \varphi_{0}}{\partial z^{2}}+\frac{\partial \varphi_{1}}{\partial z}\right)=0\right)^{*}\right) \\
& \eta_{1}=-\frac{\varphi_{1}}{\mathrm{~K}+\left(\partial \varphi_{0} / \partial z\right)} \\
& \nabla^{2} \varphi_{2}=0 \quad z<\eta_{0} \\
& n\left(\eta_{1} \frac{\partial^{2} \varphi_{1}}{\partial z \partial t}+\frac{\partial \varphi_{2}}{\partial t}\right)+2 \nabla \varphi_{0} \cdot \nabla \varphi_{2}+\left(\nabla \varphi_{1}\right)^{2}+\eta_{2} \frac{\partial}{\partial z}\left(\nabla \varphi_{0}\right)^{2}+\eta_{1}{ }^{2} \frac{\partial}{\partial z}\left(\nabla \varphi_{0} \cdot \frac{\partial}{\partial z} \nabla \varphi_{0}\right)+ \\
& +2 \eta_{1} \frac{\partial}{\partial z}\left(\nabla \varphi_{0} \cdot \nabla \varphi_{1}\right)+\mathbf{K}\left(\eta_{2} \frac{\partial^{2} \varphi_{0}}{\partial z^{2}}+\frac{\eta_{1}^{2}}{2} \frac{\partial^{3} \varphi_{0}}{\partial z^{3}}+\eta_{1} \frac{\partial^{2} \varphi_{1}}{\partial z^{2}}+\frac{\partial \varphi_{2}}{\partial z}\right)=0 \\
& \eta_{2}=-\frac{\eta_{1}\left(\frac{\eta_{1}}{2} \frac{\partial^{2} \varphi_{0}}{\partial z^{2}}+\frac{\partial \varphi_{1}}{\partial z}\right)+\varphi_{2}}{K+\left(\partial \varphi_{0} / \partial z\right)}
\end{aligned}
$$

Obviously, the procedure may be continued for higher terms.

Equation (II.7) for the $\varphi_{0}$ are, as expectable, the exact equations of steady flow.

Equations (II.8) and (II.9) are the linearized equations for first and second order terms, posed on the steady $z=\eta_{0}$ free surface.

In two important particular cases the equations (II.8) and (II.9) may be substantially simplified:

a) When the steady unperturbed state is the uniform flow (Fig. $1 a$ ).

In this case it is appropriate to transfer the equations (II.7), (II.8) and (II.9) to the axis X, Y, Z of Figure 1 . Hence, by the transformation:

$$
\mathrm{X}=x \cos \alpha-z \sin \alpha \quad \mathrm{Y}=y \quad Z=x \sin \alpha+z \cos \alpha
$$

we obtain for the different approximations:

$$
\begin{gathered}
\varphi_{0}=\mathrm{K} \sin \alpha \mathrm{X} \quad \eta_{0}=0 \quad \nabla^{2} \varphi_{1}=0 \\
n \frac{\partial \varphi_{1}}{\partial t}+\mathrm{K} \sin \alpha \frac{\partial \varphi_{1}}{\partial \mathrm{X}}+\mathrm{K} \cos \alpha \frac{\partial \varphi_{1}}{\partial Z}=0 \\
\eta_{1}=-\frac{1}{\mathrm{~K}} \frac{\varphi_{1}}{\cos \alpha} \\
\nabla^{2} \varphi_{2}=0 \quad Z<0 \\
\text { on } \mathbf{Z}=0 \\
\frac{\partial \varphi_{2}}{\partial t}+\mathrm{K} \sin \alpha \frac{\partial \varphi_{2}}{\partial \mathrm{X}}+\mathrm{K} \cos \alpha \frac{\partial \varphi_{2}}{\partial Z}=-n \eta_{1} \frac{\partial}{\partial t}\left(-\frac{\partial \varphi_{1}}{\partial \mathrm{X}}-\sin \alpha+\frac{\partial \varphi_{1}}{\partial Z} \cos \alpha\right)-
\end{gathered}
$$$$
\left.\begin{array}{c}
-\left[\left(\frac{\partial \varphi_{1}}{\partial X}\right)^{2}+\left(\frac{\partial \varphi_{1}}{\partial Y}\right)^{2}+\left(\frac{\partial \varphi_{1}}{\partial Z}\right)^{2}\right]-K \eta_{1}\left(-\sin ^{2} \alpha \frac{\partial^{2} \varphi_{1}}{\partial X^{2}}+\cos ^{2} \alpha \frac{\partial^{2} \varphi_{1}}{\partial Z^{2}}\right) \\
\eta_{2}=-\frac{\eta_{1}\left(-\frac{\partial \varphi_{1}}{\partial X} \sin \alpha+\frac{\partial \varphi_{1}}{\partial Z} \cos \alpha\right)+\varphi_{2}}{K \cos \alpha}
\end{array}\right\} \text { on } Z=\eta_{0}
$$

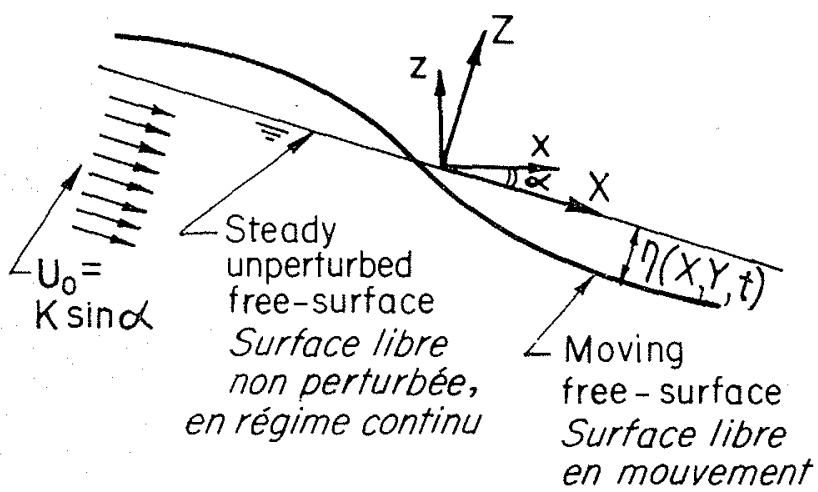

(a)

A moving free-surface. The steady unperturbed state is: a) the uniform flow. b) the rest.

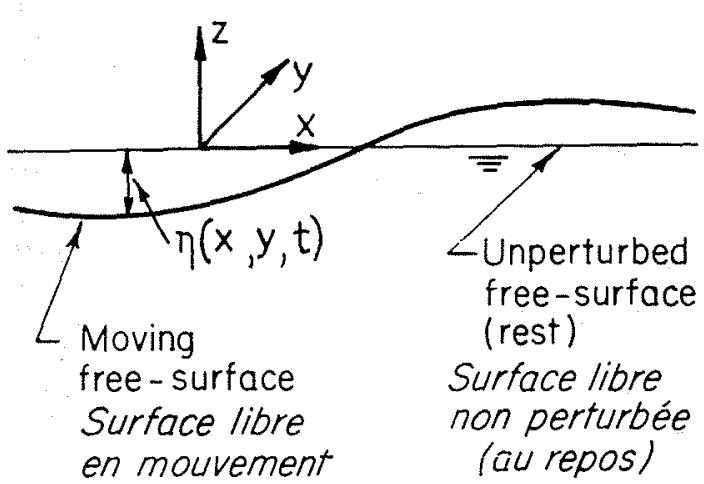

(b)

Une surface libre en mouvement. Le régime continu non perturbé comporte: a) un écoulement uniforme; b) l'eau au repos.

( ${ }^{\star}$ The symbolic notation has the meaning:

$$
\nabla \varphi_{0} \cdot \nabla \varphi_{1}=\frac{\partial \varphi_{0}}{\partial x} \frac{\partial \varphi_{1}}{\partial x}+\frac{\partial \varphi_{0}}{\partial y} \frac{\partial \varphi_{1}}{\partial y}+\frac{\partial \varphi_{0}}{\partial z} \frac{\partial \varphi_{1}}{\partial z}, \text { etc. }
$$




\section{G. DAGAN}

b) An even more particular case is that of initial rest, i.e. the case $(a)$ with $\alpha=0$, (Fig. $1 \mathrm{~b}$ ). It therefore reduces, by making $\alpha=0$ in equations (II.11) (II.12) and (II.13) to:

$$
\begin{aligned}
& \varphi_{0} \equiv 0 \\
& \nabla^{2} \varphi_{1}=0 \quad z<0 \\
& \left.\begin{array}{rl}
n \frac{\partial \varphi_{1}}{\partial t}+\mathrm{K} \frac{\partial \varphi_{1}}{\partial z} & =0 \\
\eta_{1} & =-\frac{\varphi_{1}}{\mathrm{~K}}
\end{array}\right\} \text { on } z=\eta_{0} \\
& \nabla^{2} \varphi_{2}=0 \quad z<0 \\
& n \frac{\partial \varphi_{2}}{\partial t}+\mathrm{K} \frac{\partial \varphi_{2}}{\partial z}=\frac{\varphi_{1}}{\mathrm{~K}} \frac{\partial}{\partial z}\left(n \frac{\partial \varphi_{1}}{\partial t}+\mathrm{K} \frac{\partial \varphi_{1}}{\partial z}\right)-\left[\left(\frac{\partial \varphi_{1}}{\partial \mathrm{X}}\right)^{2}+\left(\frac{\partial \varphi_{1}}{\partial \mathrm{Y}}\right)^{2}+\left(\frac{\partial \varphi_{1}}{\partial \mathrm{Z}}\right)^{2}\right] \\
& \eta_{2}=\frac{1}{K^{2}}-\frac{\partial \varphi_{1}}{\partial z} \frac{\varphi_{2}}{K}
\end{aligned}
$$

Obviously, the steady state is a particular case for which all the derivatives in respect to $t$ vanish.

Some problems of unsteady bidimensional flow have been solved by the first approximation (equations II.15) by Polubarinova Kotchina (1959), Beliacova (1955), Galin (1959), Meyer (1955), and for steady flow by Kirkham (1958) and Dagan (1964).

\section{An example of expansion in small parameter of an exact solution}

The approximation based on expansion on a small parameter exposed previously has a formal character, unless the convergence of the series (II.1) is proved.

A general proof of the convergence will ensure the validity of the expansion, on one side, and on the other side, may constitute an existence and uniqueness proof for the solution of the original nonlinear problem.

Unfortunately, beyond a simple case studied by Priazinskaia (1955) (by other means than the approximative method discussed here), there are no such proofs for problems of unsteady free-surface flow in porous media.

In this chapter the converging character of the expansion in a small parameter will be shown in a particular case of an already exactly solved problem.

For this purpose let us consider the steady bidimensional flow towards an horizontal array of equidistant sinks of equal strength Q, supplied by a uniform ascendent current at infinity 1 . With the notations of Figure 2 it is cbvious that $\mathrm{Q}=2 \mathrm{al}$.

Tile qualitative analogy between the free-surface shape and the standing waves is obvious.

The solution reproduced below was derived by van Deemter (see Schilfgaarde, Kirkham and Frevert, [1956]). Due to symmetry, the flow is investigated only in the strip SPORS (Fig. $2 a$ ).

With $\mathrm{Z}=\mathrm{X}+i \mathrm{Y}\left(^{*}\right)$ a complex variable and $\Omega=\varphi+i \psi$ the complex potential, the relations:

and:

$$
\Omega=\mathrm{K} c+\frac{a l}{\pi} \ln \frac{\mathrm{T}^{2}-1}{\mathrm{~T}^{2}-(1+\beta)^{2}}
$$

$$
Z=\alpha+i c+i \frac{a}{\pi}\left(\ln \frac{\mathrm{T}-1-\beta}{\mathrm{T}+1+\beta}+\frac{2 \mathrm{~L}}{\mathrm{~K}} \ln \frac{\mathrm{T}+1}{\mathrm{~T}+1+\beta}\right)
$$

map the $Z$ and $\Omega$ regions of flow (Fig. $2 a$ and $2 b$ ) onto an auxiliary $\mathrm{T}=s+$ ir plane (Fig. $2 c$ ).

The relations (III.1) and (III.2) contain all the elements of the solution. K-as ordinary-is the hydraulic conductivity; $a, b, c$ are characteristic dimensions (Fig. 2); $\beta$-is an auxiliary constant and is determined, for instance, by the given pressure in a certain point.

From (III.1) and (III.2) the following relations result:

$$
\begin{aligned}
\frac{\pi c}{a} & =\ln \frac{2+\beta}{\beta}+2 \frac{l}{\mathrm{~K}} \ln \frac{2+\beta}{2} \\
\frac{\pi b}{a} & =\ln \frac{2+\beta}{\beta}+2 \frac{l}{\mathrm{~K}} \ln \frac{2+\beta}{2(1+\beta)} \\
\frac{\pi(c-b)}{\mathrm{K}} & =\frac{2 l}{\mathrm{~K}} \ln (1+\beta)
\end{aligned}
$$

The purpose of the following derivations is to demonstrate that $\Omega$, and consecuently $\varphi$, can be expanded in a convergent series of the powers of a little parameter $\varepsilon$, while the consecutive terms of the expansion $\Omega_{0}$, $\Omega_{1}$, $\Omega_{2} \ldots$ are determined in a rectangular strip in the $Z$-plane, where the free-surface is replaced by a horizontal segment (according to equation II.15).

$\left.{ }^{*}\right)$ In this chapter $Z$ will signify a complex variably, $Y$ the ordinate and $X$ the absciss. To avoid confusion with the notation of the remaining chapters, capital symbols will be used. 


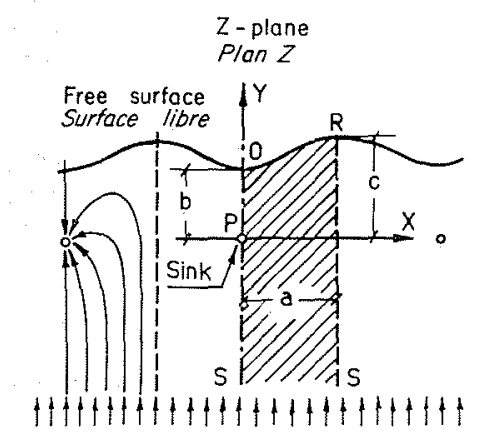

(a) $\quad l=\frac{Q}{2 a}$

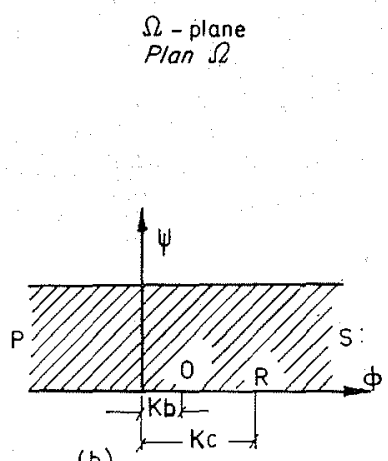

(b)

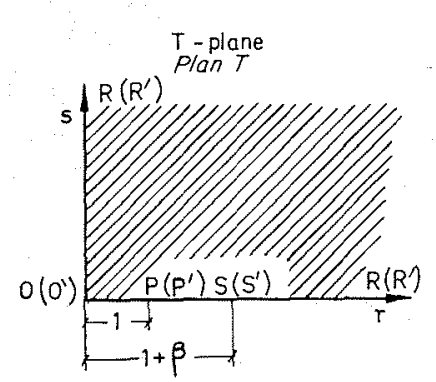

(c)

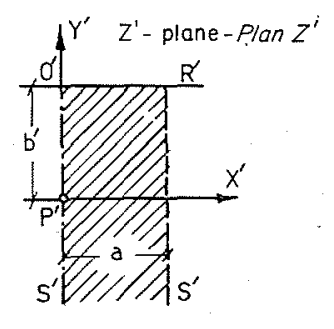

(d)

The steady bidimensional deep flow towards an array of sinks.

/2/

L'écoulement permanent plan et profond vers un ensemble de puits.

Let the strip $S^{\prime} \mathrm{P}^{\prime} \mathrm{O}^{\prime} \mathrm{R}^{\prime} \mathrm{S}^{\prime}$ be the region of flow, in which the approximate solution will be derived, in the new $Z^{\prime}=\mathrm{X}^{\prime}+i \mathrm{Y}^{\prime}$ plane (Fig. $2 d$ ). This region is mapped onto the same auxiliary $\mathrm{T}$ plane (Fig. $2 e$ ) by:

$$
Z^{\prime}=a+i b^{\prime}+i \frac{a}{\pi} \ln \frac{\mathrm{T}-1-\beta^{\prime}}{\mathrm{T}+1+\beta^{\prime}}
$$

where $b^{\prime}$ - the elevation of the straight line replacing the free-surface in the approximate solution-is taken so that $\beta^{\prime}=\beta$. This requires that:

$$
b^{\prime}=\frac{a}{\pi} \ln \frac{2+\beta}{\beta}
$$

Now, the images of the points $S^{\prime}, \mathrm{P}^{\prime}, \mathrm{O}^{\prime}, \mathrm{R}^{\prime}$, and $\mathrm{S}, \mathrm{P}, \mathrm{O}, \mathrm{R}$ in the $\mathrm{T}$ plane will be identical.

The comparison between (III.3), (III.4) and (III.7) show that $b<b^{\prime}<c$.

Let $(l / \mathrm{K})=\varepsilon$ be taken as a small parameter. The relations (III.3), (III.4) and (III.7) show that for $\varepsilon \rightarrow 0$, $Z \rightarrow Z^{\prime}$ and $(c-b) \rightarrow 0$ i. e. for the vanishing small parameter the flow region degenerates into the non perturbed scheme and the maximum free-surface drop $(c-b)$ tends to zero.

Introducing the new dimensionless variables:

$$
\begin{aligned}
& \bar{Z}=\pi \frac{Z-a-i b^{\prime}}{a} \\
& \overline{\mathrm{Z}}^{\prime}=\pi \frac{Z^{\prime}-a-i b^{\prime}}{a} \\
& \bar{\Omega}=\pi \frac{\Omega-\mathrm{K} b^{\prime}}{\mathrm{K} a}
\end{aligned}
$$

by which the origin is transferred to $\mathrm{R}^{\prime}$, the relations (III.1), (III.2) and (III.6) transform into:

$$
\begin{aligned}
& \bar{Z}=i\left(\ln \frac{\mathrm{T}-1-\beta}{\mathrm{T}+1+\beta}+2 \varepsilon \ln \frac{(2+\beta)(\mathrm{T}+1)}{2(\mathrm{~T}+1+\beta)}\right) \\
& \bar{\Omega}=\varepsilon \ln \frac{(2+\beta)^{2}\left(\mathrm{~T}^{2}-1\right)}{4\left[\mathrm{~T}^{2}-(1+\beta)^{2}\right]} \\
& \overline{Z^{\prime}}=i \ln \frac{\mathrm{T}+1+\beta}{\mathrm{T}-1-\beta}
\end{aligned}
$$

which map the new variables on the T-plane. From (III.11) the mapping of $T$ on $Z^{\prime}$ is given by:

$$
\mathrm{T}=-i(1+\beta) \operatorname{cotan} \frac{\overline{Z^{\prime}}}{2}
$$

From (III.9), (III.11) and (III.12):

$$
\bar{Z}-\bar{Z}^{\prime}=\varepsilon 2 i \ln \frac{(2+\beta)\left[1-i(1+\beta) \operatorname{cotan}\left(\bar{Z}^{\prime} / 2\right)\right]}{2(1+\beta)\left[1-i \operatorname{cotan}\left(\bar{Z}^{\prime} / 2\right)\right]}
$$

The relation (III.13) maps the flow region $\bar{Z}$ onto the plain of the approximate solution $\bar{Z}^{\prime}$. It can be written in an abbreviated form as:

$$
\bar{Z}=Z^{\prime}+\varepsilon f\left(\bar{Z}^{\prime}\right)
$$

\footnotetext{
* N.D.L.R. - Le surlignage appliqué aux symboles $Z, Z^{\prime}$ et $\Omega$ ne signifie pas ici que ce sont des yaleurs moyennes, mais que ce sont des variables sans dimensions.
} 
The function $f\left(\bar{Z}^{\prime}\right)$ is regular in the strip $S^{\prime} P^{\prime} O^{\prime} R^{\prime} S$, its maximum is reached for:

$$
\left|f\left(\bar{Z}^{\prime}\right)\right|_{\bar{Z}^{\prime}=0}=2 \ln \frac{2+\beta}{2}
$$

In each point of the $\bar{Z}^{\prime}$ plane the equation (III.13) defines a circle of radius $\left|\bar{Z}-\bar{Z}^{\prime}\right|$. The function $\bar{\Omega}\left(\overline{Z^{\prime}}\right)$ can be expanded in Taylor series into such a circle since $\bar{\Omega}\left(\bar{Z} \bar{Z}^{\prime}\right)$ is analytic in the half-strip $S^{\prime} P^{\prime} O^{\prime} R^{\prime} S^{\prime}$ and takes real values on the upper boundary $\mathrm{Y}^{\prime}=0$; hence, it may be analitically continued beyond this boundary and obviously beyond $\mathrm{S}^{\prime} \mathrm{R}^{\prime}$ (Fig. $2 d$ ).

Hence, the expansion:

$$
\bar{\Omega}\left[\bar{Z}^{\prime}+\varepsilon f\left(\bar{Z}^{\prime}\right)\right]=\bar{\Omega}\left(\bar{Z}^{\prime}\right)+\varepsilon f\left(\bar{Z}^{\prime}\right) \frac{d \bar{\Omega}}{d \bar{Z}^{\prime}}+\frac{\varepsilon^{2}}{2} f\left(\bar{Z}^{\prime}\right)^{2} \frac{d^{2} \bar{\Omega}}{d \bar{Z}^{\prime 2}}+\ldots
$$

is convergent in each point of the $\bar{Z}^{\prime}$-plane, and according to (III.13) represents the $\bar{\Omega}(\bar{Z})$.

After some computations, the first two terms look:

$$
\bar{\Omega}(\bar{Z})=\varepsilon\left\{\begin{array}{c}
2 \ln \frac{2+\beta}{4(1+\beta)}+ \\
+\ln \left[1+\beta(2+\beta) \cos ^{2} \frac{\bar{Z}^{\prime}}{2}\right]
\end{array}\right\}-\varepsilon^{2} \frac{2 i \beta(2+\beta) \sin \bar{Z}^{\prime}}{1+\beta(2+\beta) \cos ^{2}\left(\bar{Z}^{\prime} / 2\right)} \ln \frac{(2+\beta)\left[2+\beta\left(1+e^{-i \bar{Z})}\right]\right.}{4(1+\beta)}+\ldots
$$

$\varphi$ expansion will result from:

$$
\varphi\left(\mathrm{X}^{\prime}, \mathrm{Y}^{\prime}\right)=\mathcal{R} \Omega\left(Z^{\prime}\right)
$$

and will be obviously a convergent series in $\varepsilon$.

In this case the convergence is assured for arbitrary $\varepsilon$, since $b, c$ and $b^{\prime}$ have been taken according to the exact solution.

\section{Two applications of the second order theory}

The solution of problems of free-surface flow in porous mediums by second order approximation will be illustrated by two simple examples of bidimensional flow.

a) The steady Free-surface flow Through a POROUS DaM

This case has been studied by Muskat (1937) and Polubarinova-Kotchina (1952) by exact methods.

For the approximate solution, let us take $\varepsilon=(\Delta \mathrm{H} / \mathrm{L}$ ) as a small parameter (Fig. $3 a$ ). $\varphi$ and $\eta$ are expanded up to third order terms as following:

$$
\begin{aligned}
& \varphi=\varepsilon \varphi_{1}+\varepsilon^{2} \varphi_{2} \\
& \eta=\varepsilon \eta_{1}+\varepsilon^{2} \eta_{2}
\end{aligned}
$$

since the non-perturbed state corresponding to $\varepsilon=0(\Delta=0)$ is the rest.

The boundary conditions for $\varphi, \varphi_{1}$ and $\varphi_{2}$ are chosen according to (I.2), (II.15) and (II.16) ( ${ }^{\star}$ ) and given in Figures $3 a, 3 b$ and $3 c$.

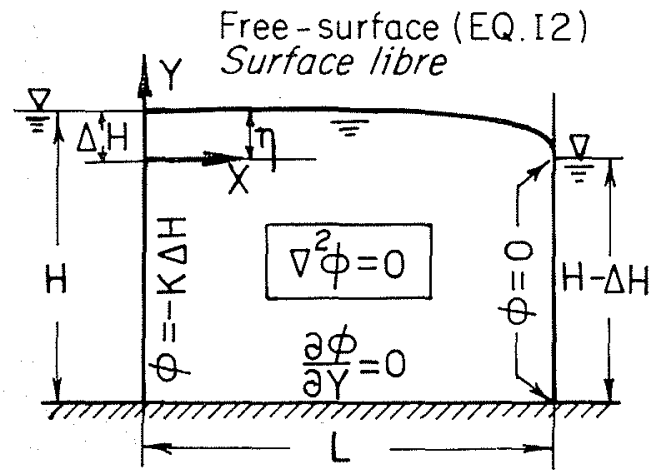

(a)

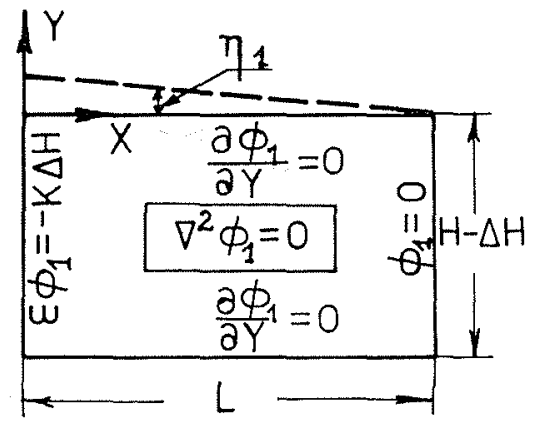

(b)

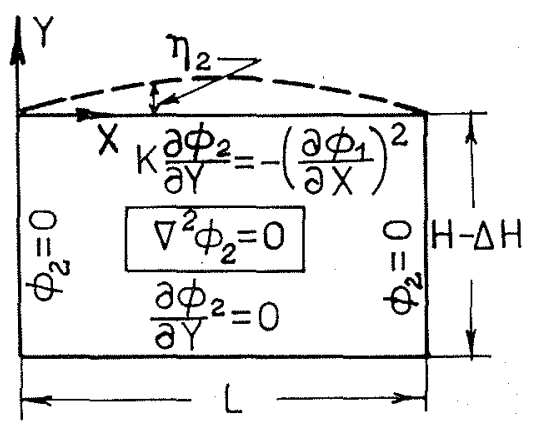

(c)

Seepage through a dam; boundary conditions for : a) $\varphi, b) \varphi, c) \varphi a$.

3. Percolation par un barrage; conditions aux limites correspondant $\dot{a}:$ a) $\varphi$, b) $\varphi$, , c) $\varphi$. 
The solution for $\varphi_{1}$ is quite simple:

$$
\left.\begin{array}{l}
\varphi_{1}=-\frac{\mathrm{K} \Delta \mathrm{H}[1-(\mathrm{X} / \mathrm{L})]}{\varepsilon}=-\mathrm{KL}[1-(\mathrm{X} / \mathrm{L})] \\
\eta_{1}=\mathrm{L}(1-\mathrm{X} / \mathrm{L})
\end{array}\right\}
$$

and represents the uniform flow through the confined rectangle of Figure $3 b$.

For $\varphi_{2}(\mathrm{Fig} .3 \mathrm{c}$ ) a Fourier series representation is suitable:

$$
\varphi_{2}=\sum_{1}^{\infty} \operatorname{An} \cosh \frac{n \pi(\mathrm{Y}+\mathrm{H}-\Delta \mathrm{H})}{\mathrm{L}} \sin \frac{n \pi \mathrm{X}}{\mathrm{L}}
$$

$\varphi_{2}$, in this form, satisfies Laplace equation and all boundary conditions excepting on $\mathrm{Y}=0$.

On this boundary:

$$
\frac{\partial \varphi_{2}}{\partial \mathbf{Y}}=-\frac{1}{K}\left(\frac{\partial \varphi_{1}}{\partial X}\right)^{2}=-\mathbf{K}
$$

Computing An from (IV.5), it is finally obtained:

$$
\begin{aligned}
& \varphi_{2}=-\frac{4 \mathrm{KL}}{\pi^{2}} \sum_{0}^{\infty} \frac{\cosh \frac{\pi(2 n+1)(\mathrm{Y}+\mathrm{H}-\Delta \mathrm{H})}{\mathrm{L}} \sin \frac{\pi(2 n+1) \mathrm{X}}{\mathrm{L}}}{(2 n+1)^{2} \sinh \frac{\pi(2 n+1)(\mathrm{H}-\Delta \mathrm{H})}{\mathrm{L}}} \\
& \eta_{2}=-\left.\frac{\varphi_{2}}{\mathrm{~K}}\right|_{\mathrm{X}=0}=\frac{4 \mathrm{~L}}{\pi^{2}} \sum_{0}^{\infty} \frac{\operatorname{cotanh} \frac{\pi(2 n+1)(\mathrm{H}-\Delta \mathrm{H})}{\mathrm{L}} \sin \frac{\pi(2 n+1) \mathrm{X}}{\mathrm{L}}}{(2 n+1)^{2}}
\end{aligned}
$$

Consequently, by (IV.1) the final expression of $\eta$ is:

$$
\frac{\eta(\mathrm{X})}{\mathrm{L}}=\left(1-\frac{\mathrm{X}}{\mathrm{L}}\right) \frac{\Delta \mathrm{H}}{\mathrm{L}}+\frac{4}{\pi^{2}}\left(\frac{\Delta \mathrm{H}}{\mathrm{L}}\right)^{2} \sum_{0}^{\infty} \frac{\operatorname{cotanh} \frac{\pi(2 n+1)(1-\Delta \mathrm{H})}{\mathrm{L}} \sin \frac{\pi(2 n+1) \mathrm{X}}{\mathrm{L}}}{(2 n+1)^{2}}
$$

\section{The evaluation of the discharge}

The exact expression for the flux through a vertical section is given by:

By the expansions (IV.1) it becomes:

$$
\mathrm{Q}=\int_{0}^{\mathrm{H}-\Delta \mathrm{H}+\eta} \frac{\partial \varphi}{\partial \mathrm{X}} d \mathrm{Y}
$$

$\mathbf{Q}=\int_{0}^{\mathrm{H}-\Delta \mathrm{H}+\varepsilon \eta_{1}+\varepsilon^{2} \eta_{2}}\left(\varepsilon \frac{\partial \varphi_{1}}{\partial \mathrm{X}}+\varepsilon^{2} \frac{\partial \varphi_{2}}{\partial \mathrm{X}}\right) d \mathbf{Y}=\varepsilon \int_{0}^{\mathrm{H}-\Delta \mathrm{H}} \frac{\partial \varphi_{1}}{\partial \mathrm{X}} d \mathbf{Y}+\varepsilon^{2}\left[\left.\eta_{1} \frac{\partial \varphi_{1}}{\partial \mathrm{X}}\right|_{\mathrm{Y}=0}+\int_{0}^{\mathrm{H}-\Delta \mathrm{H}} \frac{\partial \varphi_{9}}{\partial \mathrm{X}} d \mathrm{Y}\right]+0\left(\varepsilon^{3}\right)$

From the solutions of $\varphi_{1}$ and $\varphi_{2}$ (IV.3) and (IV.6) the discharge, up to third order terms, resulting from (IV.10) is:

$$
\mathrm{Q}=\varepsilon \mathrm{K}(\mathrm{H}-\Delta \mathrm{H})+\varepsilon^{2} \frac{\mathrm{KL}}{2}=\mathrm{K} \frac{\mathrm{H}^{2}-(\mathrm{H}-\Delta \mathrm{H})^{2}}{2 \mathrm{~L}}
$$

However, $Q$ from (IV.11) is the exact value of the discharge (Polubarinova-Kotchina, 1952).

This striking fact suggests that the higher terms in $Q$ expansion cancel.

Comparison with Dupuit solution

In the particular case in which not only $\Delta \mathrm{H} / \mathrm{L}$ is small, but $\mathrm{H} / \mathrm{L}$ also (shallow depth flow) the solution (IV.6) for $\varphi_{2}$ may be replaced by the more simple one derived by Polubarinova-Kotchina (1952) for an infinite long strip:

$$
\varphi_{2}=\frac{\mathrm{K}}{2(\mathrm{H}-\Delta \mathrm{H})}\left[\left(\mathrm{X}-\frac{\mathrm{L}}{2}\right)^{2}-(\mathrm{Y}+\mathrm{H}-\Delta \mathrm{H})^{2}\right]+\text { const }
$$

This solution cannot satisfy the condition $\varphi_{2}=0$ at $X=0$ and $X=L$, but is able to represent fairly $\varphi_{2}$ in the central portion of the strip $(\mathrm{X} \approx \mathrm{L} / 2)$. With $\varphi_{2}=0$ at $\mathrm{X}=0, \mathrm{~L}$ and $\mathrm{Y}=0$, (IV.12) becomes:

$$
\varphi_{2}=\frac{K}{2(H-\Delta H)}[X(X-L)-Y(Y+2 H-2 \Delta H)]
$$

The solution based on Dupuit assumption for the seepage through a dam is:

$$
\mathrm{H}^{2}-(\mathrm{H}-\Delta \mathrm{H}+\eta)^{2}=\frac{2 \mathrm{Q} x}{\mathrm{~K}}
$$




\section{G. DAGAN}

By (IV.11) and some transformations (IV.14) becomes:

$$
\eta=\Delta \mathrm{H}-\mathrm{H}+\mathrm{H}\left[1-\frac{\Delta \mathrm{H}}{\mathrm{H}}\left(2-\frac{\Delta \mathrm{H}}{\mathrm{H}}\right) \frac{\mathrm{X}}{\mathrm{L}}\right]^{1 / 2}
$$

Supposing $\Delta \mathrm{H} / \mathrm{H}$ small, the square root may be expanded into a series and (IV.15) yields:

$$
\eta / \mathrm{L}=\frac{\Delta \mathrm{H}}{\mathrm{L}}\left(1-\frac{\mathrm{X}}{\mathrm{L}}\right)+\frac{1}{2}\left(\frac{\Delta \mathrm{H}}{\mathrm{L}}\right)^{2} \frac{\mathrm{X}}{\mathrm{H}}\left(1-\frac{\mathrm{X}}{\mathrm{L}}\right)+0\left(\varepsilon^{3}\right)
$$

Hence, the term in $\varepsilon^{2}$ by the linearized solution for $\eta / L$ is according to (IV.13):

$$
\eta_{2} / L=-\left.\frac{\varphi_{2}}{\mathrm{~K}}\right|_{\mathrm{Y}=0}=\frac{\mathrm{X}}{2(\mathrm{H}-\Delta \mathrm{H})}\left(1-\frac{\mathrm{X}}{\mathrm{L}}\right)
$$

and the similar one from the solution based on Dupuit assumption according to (IV.16) is:

$$
\eta_{2}(\text { Dupuit }) / L=\frac{X}{2 H}\left(1-\frac{X}{L}\right)
$$

$\eta_{2} / \mathrm{L}$ from (IV.17) and (IV.18) are very closed if $(\Delta \mathrm{H} / \mathrm{H}) \ll 1$.

In conclusion, for:

$$
\frac{\Delta H}{\mathrm{~L}} \ll 1, \mathrm{H} / \mathrm{L} \ll 1 \text { and } \frac{\Delta \mathrm{H}}{\mathrm{H}} \ll 1
$$

the linearized theory and the non-linear one based on Dupuit assumptions give close results up to the third order terms of the free-surface equation.

Deep flow through a dam

When $\frac{H}{L}>1$, the cotanh $\frac{\pi(2 n+1)(H-\Delta H)}{L}$ in equation (IV.8) is very close to 1 . Hence, for deep flow the free-surface shape is no more influenced by the dam height, and (IV.8) becomes:

$$
\frac{\eta(\mathrm{X})}{\mathrm{L}}=\left(1-\frac{\mathrm{H}}{\mathrm{L}}\right) \frac{\Delta \mathrm{H}}{\mathrm{L}}+\left(\frac{\Delta \mathrm{H}}{\mathrm{L}}\right)^{2} \frac{4}{\pi^{2}} \sum_{0}^{\infty} \frac{\sin [\pi(2 n+1) \mathrm{X} / \mathrm{L}]}{(2 n+1)^{2}}
$$

$\eta$ has been computed from this rapid convergent series up to ten terms by aid of a high-speed electronic computer for $\Delta \mathrm{H} / \mathrm{L}=0.1$. The results are given in Figure 4 .

b) Damping of an INITIAL COSINUSOIdAL FREE-SURFACE

Let us suppose that at $t=0$ the free-surface bounding a region of infinite depth (Fig. 5) has the shape:

$$
\eta=\varepsilon \cos \alpha \mathrm{X}
$$

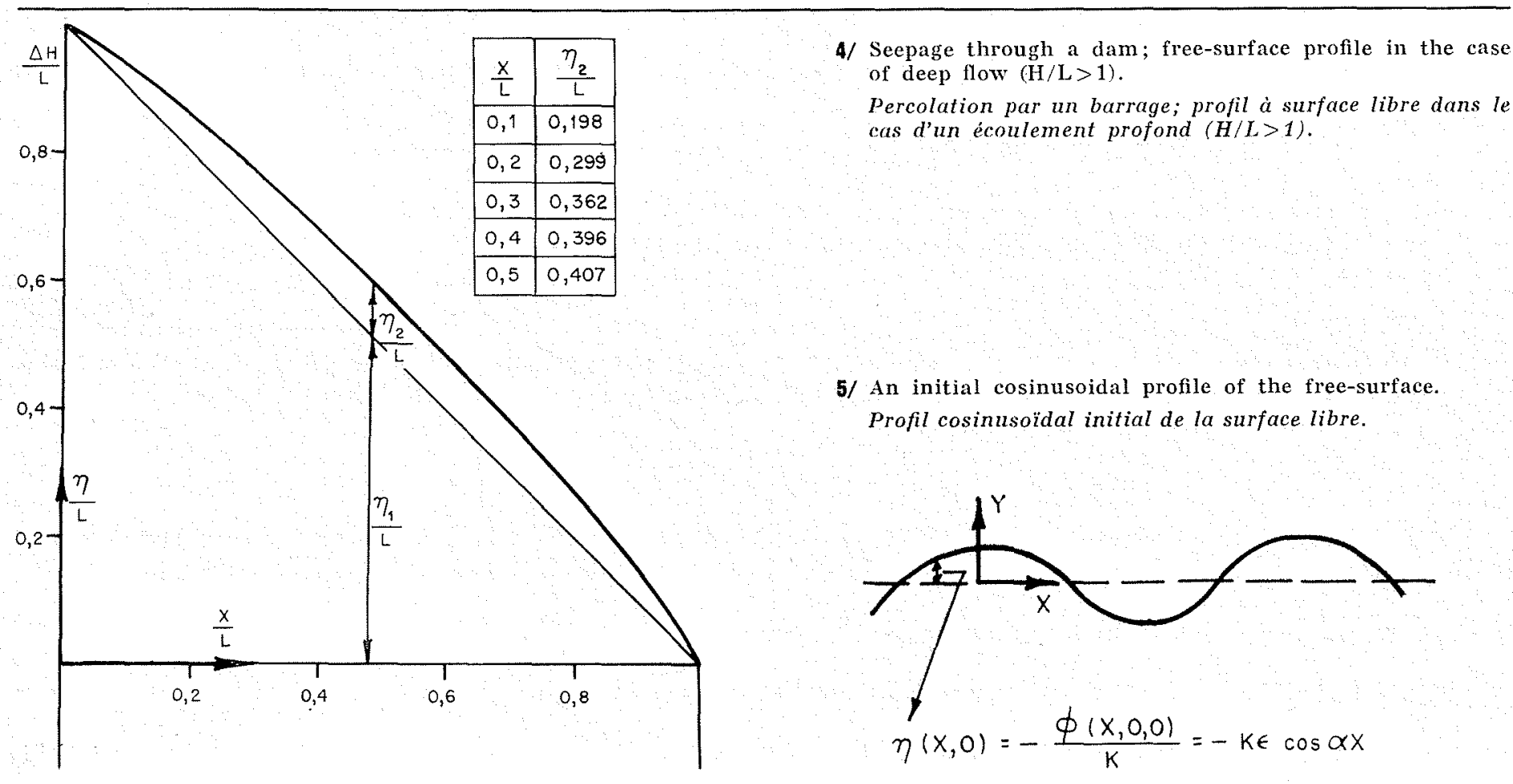


Where $\varepsilon=A \alpha, A$ being a length determining the amplitude of $\eta\left({ }^{\star}\right)$.

Up to third order terms, $\varphi$ and $\eta$ have the expansions (IV.1). According to (IV.15) $\varphi_{1}$ satisfies the conditions:

$$
\begin{array}{cc}
n \frac{\partial \varphi_{1}}{\partial t}+\mathrm{K} \frac{\partial \varphi_{1}}{\partial \mathrm{Y}}=0 & \text { on } \mathrm{Y}=0 \\
\varphi=-\mathrm{K} \eta_{1}=-\mathrm{K} \cos \alpha \mathrm{X} & \text { on } \mathrm{Y}=0 \quad \text { at } t=0
\end{array}
$$

The harmonic function, vanishing at $-\infty$, satisfying (IV.20) is:

and :

$$
\begin{aligned}
& \varphi_{1}=-\mathrm{K} e^{\alpha[\mathrm{Y}-(\mathrm{K} / n) t]} \cos \alpha \mathrm{X} \\
& \eta_{1}=e^{-\alpha(\mathrm{K} / n) t} \cos \alpha \mathrm{X}
\end{aligned}
$$

The second approximation fulfills (II.16) which becomes in the present case:

and :

$$
\mathrm{n} \frac{\partial \varphi_{2}}{\partial t}+\mathbf{K} \frac{\partial \varphi_{2}}{\partial \mathbf{Y}}=-\mathbf{K}^{2} \alpha^{2} e^{-2 \alpha(\mathrm{K} / n) t} \quad \text { on } \mathbf{Y}=0
$$

$$
\eta_{2}=\alpha \cos ^{2} \alpha \mathrm{X} e^{-2 \alpha(\mathrm{K} / n) t}-\frac{\varphi_{0}}{\mathrm{~K}}=0 \quad \text { on } \mathrm{Y}=0 \quad \text { at } t=0
$$

The appropriate solution for $\varphi_{2}$ is:

$$
\varphi_{2}=\frac{\alpha \mathrm{K}}{2} e^{-2 \alpha\left(\mathrm{K}^{2} / n\right) t}\left(1+e^{2 \alpha \mathrm{Y}} \cos ^{2} \alpha \mathrm{X}\right)
$$

Hence, from (IV.1), (II.15), (IV.21) and (IV.23), the solutions of $\varphi$ and are:

$$
\begin{aligned}
& \varphi=-\mathrm{K} e^{\alpha[\mathrm{Y}-(\mathrm{K} / n) t]} \cos \alpha \mathrm{X}+\varepsilon^{2} \frac{\alpha \mathrm{K}}{2} e^{-2 \alpha(\mathrm{K} / n) t}\left(1+\mathrm{e}^{2 \alpha \mathrm{Y}} \cos 2 \alpha \mathrm{X}\right)+0\left(\varepsilon^{3}\right) \\
& \eta=\varepsilon e^{-\alpha(\mathrm{K} / n) t} \cos \alpha \mathrm{X}+0\left(\varepsilon^{3}\right)
\end{aligned}
$$

The striking fact shown up in (IV.24) is that the free-surface profile is approximated by the first term at least up to third order.

\section{Conclusions}

It seems that the linearized theory or different orders makes possible the solution of a large class of free-surface flow problems. The great advantage of the approach derives from the possibility of using the great variety of mathematical solutions for partial derivatives equations with linear boundary conditions. It is apparent that a substantial progress may be achevied in two distinct directions: the thoroughly mathematical study of the convergence of the series expansions and the derivation of new solutions for different particular cases.

\section{List of Symbols}

$a, b, c, b^{\prime}=$ geometrical dimensions;

$\mathrm{H}=$ height;

$\Delta H=$ level difference;

$k=$ intrinsic hydraulic conductivity;

$\mathrm{K}=$ hydraulic conductivity;

$l=$ specific discharge $(\mathrm{L} / \mathrm{T})$;

$\mathrm{L}=$ length;

$\mathrm{Q}=\operatorname{discharge}\left(\mathrm{L}^{2} / \mathrm{T}\right)$;

$\alpha=$ angle;

$\beta, \beta^{\prime}=$ parameters of integration;

$\gamma=$ specific weight; $\varphi=$ velocity potential

$\frac{\partial \varphi}{\partial n}=$ normal derivative

$\varepsilon=$ small parameter;

$\mu=$ viscosity (dynamic);

$\eta=$ free-surface elevation;

$\Omega=$ complex velocity potential;

$\psi=$ stream function;

$p=$ density;

$0\left(\varepsilon^{2}\right)=$ power series in $\varepsilon$ starting with $\varepsilon^{2}$. 
Bear (J.) and Dagan (G.). - The use of the hodograph method for ground water investigations, Technion-Israel Institut of Technology, Hydraulic Laboratory, P.N. 24 (1962).

BeLiacova (V. K.). - Unsteady flow of groundwater towards an horizontal drain, Prikladnaia Matematika $i$ Mehanika, vol. 19 (1955).

DAGAN (G.). - Spacing of drains by an approximate method, Proc. A.S.C.E., Jonrn. of Dr. and Tr. Div., vol 90, No. 1 (1964).

Galin (L. A.). - Unsteady seepage of groundwater towards a narrow ditch, Prikladnaia Matematika i Mehanika, vol. $23(1959)$.

KInkham (D.). - Seepage of steady rainfall through soil into drains, Trans. Am. Geoph. Union, vol. 39, No. 5 (1958).

Mexer (R.). - Quelques résultats thériques récents concernant les écoulements des nappes d'eau souterraines, La Honille Blanche, No. 1 (1955), No. 5 (1955), No. 1 (1956).
Muskar (M.). - The flow of homogeneous fluids through porous media, McGraw-Hill, New York (1937).

Polubarinova-Kotchina (P.Ya.). - Theory of ground water movement, Governmental Publishing Office for Technical and Theoretical Literature, Moscow, (1952), (also Princeton 1962).

Polubarinova-Kotchisa (P. Ya.). - Ground water movements at water level fluctuations in a reservoir with a vertical boundary, Prikladnaia Matematika i Mehanilka, vol. 23 , No. 3 (1959).

Priazinskaya (V. O.). - - The problem of plane unsteady motion of ground waters, Prikladnaia Matematika $i \mathrm{Me}$ hanika, vol. 23 , No. 5 (1955).

Schilfgatide (J.), Kirkham (D.) and Frevert (R. K.). Physical and mathematical theories of tile and ditch drainage and their usefulness in design, Iowa, Research Bulletin, No. 436 (1956).

Résumé

\title{
Théorie linéarisée, du deuxième ordre, de l'écoulement à surface libre dans les milieux poreux
}

\author{
par G. Dagan *
}

On examine l'écoulement à surface libre d'un liquide incompressible et homogène dans un milieu poreux isotrope, homogène et indéformable.

Pour résoudre exactement un tel problème, il est nécessaire de déterminer le potentiel de vitesse, $\varphi$, comme fonction harmonique (équation I.1) respectant une condition aux limites non linéaire (équation I.2) sur la surface libre inconnue en mouvement (équation I.3).

Une méthode approchée consiste à développer les expressions, correspondant au potentiel, et à la surface libre, en une série exponentielle d'un paramètre $\varepsilon$ de faible valeur (équations II.1, II.2), semblable à l'approximation des ondes infinitésimales. On dérive ensuite les équations définissant les deux premiers termes (équations II.8, II.9). Alors que les fonctions restent harmoniques, les conditions à la surface libre deviennent linéaires, et sont posées pour une surface permanente, et a priori connue. Deux cas sont examinés en détail, d'une part, celui d'une surface permanente correspondant à un plan incliné (équations II.10-II.13, fig. $1 a$ ), et d'autre part, celui d'une surface plane et horizontale (équations II.14-II.16, fig. $1 b$ ).

On examine la convergence des développements en fonction d'un paramètre de faible valeur, pour le cas d'un écoulement plan se dirigeant vers un ensemble de puits (fig. 2), pour lequel Van Deemter avait trouvé une solution exacte. Il apparaît la possibilité de développer le potentiel complexe $\Omega$ en une série (équation III.15), convergente pour une valeur arbitraire de $\varepsilon$.

Deux exemples de solutions du deuxième ordre sont présentés

Dans le cas d'un écoulement plan permanent par un barrage, le profil de la surface libre est défini par l'équation IV.8. On montre que l'on aboutit à une évaluation exacte du débit, et que ces résultats sont voisins de celui obtenu à l'aide des hypothèses de Dupuit, à condition que l'écoulement soit peu profond (équations IV.17, IV.18). On calcule le profil de la surface libre pour le cas d'un écoulement profond (équation VI.19, fig. 4).

L'amortissement d'une surface libre initiale de profil cosinusoïdal est un exemple d'un écoulement non permanent. La solution est exprimée par l'équation IV.24. On croit pouvoir obtenir une importante classe de nouvelles solutions à l'aide de la théorie linéarisée.

* Lecturer, Hydraulic Laboratory, Technion-Israel Institute of Technology. 